\title{
A RECURSION FORMULA FOR THE COEFFICIENTS IN AN ASYMPTOTIC EXPANSION
}

\author{
by E. M. WRIGHT
}

(Received 19th August, 1958)

Mäny authors have proved results deducing an asymptotic expansion of

$$
F(z)=\sum_{n=0}^{\infty} f(n) z^{n}
$$

for large $|z|$ from the behaviour of $f(t)$, when $f(t)$ is regular in an appropriate part of the complex $t$-plane. For example, if, for some $\kappa>0$ and some $A_{m}, \alpha_{m}$,

$$
f(t)=\sum_{m=1}^{M} \frac{\kappa A_{m}}{\Gamma\left(\kappa t+\alpha_{m}\right)}+O\left(\frac{1}{\Gamma\left(\kappa t+\alpha_{m+1}\right)}\right)
$$

for all large $|t|$ such that $\mathscr{R}(t)>C$, then, as $|z| \rightarrow \infty$ in a suitable sector in the $z$-plane, we have

$$
F(z)=Z e^{Z}\left\{\sum_{m=1}^{M} A_{m} Z^{-\alpha_{m}}+O\left(Z^{-\alpha_{\mathbf{H}+1}}\right)\right\}
$$

where $Z$ is an appropriate value of $z^{1 / k}$.

Of course, the expansion (1) could be replaced by one of another form, but (1) has the merit of displaying the actual coefficients which occur in (2). So far as I am aware, the first notice of this phenomenon in special cases occurred in [1] and [6] ; the general result was found in [7] and, independently, in [2]. See also [8].

A particular case of the generalised hypergeometric function studied in [6] is

$$
G(x)=\sum_{n=0}^{\infty} g(n) x^{n},
$$

where

$$
g(t)=\prod_{r=1}^{p} \Gamma\left(t+\beta_{r}\right) / \prod_{r=0}^{q} \Gamma\left(t+\gamma_{r}\right)
$$

and $q \geqslant p \geqslant 0$. If we write $\kappa=q+1-p$ and

$$
\vartheta=\sum_{r=1}^{p} \beta_{r}-\sum_{r=0}^{q} \gamma_{r}+\frac{1}{2}(\kappa+1)
$$

we can deduce from the well-known asymptotic expansion of the $\Gamma$-function that

$$
g(t)=a\left(\kappa^{\kappa}\right)^{t}\left\{\sum_{m=0}^{M} \frac{\kappa c_{m}}{\Gamma(\kappa t-\vartheta+m+1)}+O\left(\frac{1}{\Gamma(\kappa t-\vartheta+M+2)}\right)\right\}
$$

for large $|t|$ and $|\arg t|<\pi-\epsilon$, where $\epsilon>0, c_{0}=1$ and $a=(2 \pi)^{i-i \kappa} \kappa^{-1-\vartheta}$. It then follows that, in a suitable sector of the $x$-plane enclosing the positive half of the real axis, we have

$$
G(x)=a X^{\vartheta} e^{X}\left\{\sum_{m=0}^{M} c_{m} X^{-m}+O\left(X^{-M-1}\right)\right\}
$$

where $X=\kappa x^{1 / \kappa}$. 
Recently Riney $[4,5]$ has found two linear recurrence relations satisfied by the $c_{m}$. In the one, $c_{m}$ is given in terms of all the preceding $c_{n}$; in the other, in terms of the preceding $q+1$ terms of the sequence. His method in each case depends on fairly elaborate manipulations of $g(t)$. My purpose here is to point out that $G(x)$ and its asymptotic expansion alike satisfy a simple differential equation and that from this a finite recurrence formula for the $c_{m}$ can be deduced fairly simply.

We write $\theta=x d / d x$ and

$$
P(t)=\prod_{r=1}^{p}\left(t+\beta_{r}\right), \quad Q(t)=\prod_{r=0}^{q}\left(t+\gamma_{r}\right)
$$

and note that

$$
Q(t) g(t+1)=P(t) g(t)
$$

Aguin

$$
\begin{aligned}
x P(\theta) G(n) & =\sum_{n=0}^{\infty} P(n) g(n) x^{n+1} \\
& =\sum_{n=0}^{\infty} Q(n) g(n+1) x^{n+1} \\
& =Q(\theta-1) \sum_{n=0}^{\infty} g(n+1) x^{n+1} \\
& =Q(\theta-1)\{G(x)-g(0)\} .
\end{aligned}
$$

Hence, if $R(t)^{\circ}=Q(t-1)-x P(t)$, we have

$$
R(\theta) G(x)=Q(-1) g(0) .
$$

This is the linear differential equation of the $(q+1)$-th order satisfied by $G(x)$.

We need not appeal to the general theory of asymptotic solutions of differential equations to see that (4) is satisfied asymptotically for (say) large positive $X$, if the right-hand side of (3) is substituted for $G(x)$. For $\theta G(x)$ is a function of the same form as $G(x)$ and so has a similar asymptotic expansion.

Let us write $\phi=X(d / d X)=\kappa \theta$,

$$
T(t)=\prod_{r=0}^{q}\left(t-\kappa+\kappa \gamma_{r}+\vartheta\right), \quad U(t)=\prod_{r=1}^{p}\left(t+\kappa \beta_{r}+\vartheta\right)
$$

and

$$
S(t)=T^{\prime}(t-\vartheta)-X^{q+1-p} U(t-\vartheta)=\kappa^{a+1} R(t / \kappa)
$$

$1 \mathrm{y}(4)$ and (5),

$$
S(\phi) e^{X} \sum_{m=0}^{M} c_{m} X^{\vartheta-m}=O\left(X^{\vartheta+q-M} e^{X}\right)
$$

for any positive $M$. Now

$$
\phi X^{j} e^{X}=X^{j}(\phi+j) e^{X}
$$

and so

$$
S(\phi) e^{X} X^{\vartheta-m}=X^{\vartheta-m} S(\phi+\vartheta-m) e^{X}
$$


Since $T(t)$ is a polynomial in $t$ of degree $q+1$, we have

$$
T(t-m)=\sum_{s=0}^{q+1} T_{s}(-m) t(t-1) \ldots(t-s+1),
$$

where

$$
T_{s}(-m)=\sum_{r=0}^{s} \frac{(-1)^{s-r} T(r-m)}{r !(s-r) !}=\frac{\Delta^{s} T(-m)}{s !}
$$

in the usual notation of the difference calculus. Hence

$$
T(\phi-m)=\sum_{s=0}^{q+1} T_{s}(-m) X^{s}(d / d X)^{s}
$$

and

$$
e^{-X} T(\phi-m) e^{X}=\sum_{s=0}^{q+1} T_{s}(-m) X^{s}
$$

Similarly

$$
e^{-X} U(\phi-m) e^{X}=\sum_{8=0}^{p} U_{s}(-m) X^{*}
$$

Hence

$$
e^{-X} S(\phi+\vartheta-m) e^{X}=X^{a+1}\left\{\sum_{s=0}^{q+l} T_{a+1-s}(-m) X^{-s}-\sum_{s=0}^{p} U_{p-s}(-m)^{-} X^{s}\right\}
$$

and so, by (6) and (7),

$$
\sum_{s=0}^{q+1} T_{a+1-s}(s-m) c_{m-s}-\sum_{s=0}^{p} U_{p-s}(s-m) c_{m-s}=0
$$

where $c_{n}=0$, when $n<0$.

We shall see later that

$$
\begin{gathered}
T_{a+1}(-m)-U_{p}(-m)=0, \ldots \\
T_{q}(-m)-U_{p-1}(-m)=-\kappa m .
\end{gathered}
$$

Hence, if we replace $m$ by $m+1$ and $s$ by $s+1$ in (8), we have

$$
\kappa m c_{m}=\sum_{s=1}^{q} T_{q-s}(s-m) c_{m-s}-\sum_{s=1}^{p-1} U_{p-s-1}(s-m) c_{m-s},
$$

where the second sum is empty if $p=0$ or 1 . This is the recurrence relation required.

If the largest $m$ for which we wish to calculate $c_{m}$ is of about the size of $q$, the coefficients in (11) can be most easily calculated by evaluating $T(t)$ for $t=q-1, q-2, \ldots,-m$ and then differencing these values up to $(q-1)$ times. If $m$ is large compared with $q$, we remark that

$$
T_{a-s}(s-m)=\sum_{r=0}^{s+1}(-1)^{s+1-r} T_{a+1-r}(0)(q+1-r) !(m-r) ! /\{(m-s-1) !(q-s) !(s+1-r) !\}
$$

so that we need only calculate $T_{s}(0)$ (by differencing) for $s=0, \ldots, q+1$. Similarly

$$
U_{p-s-1}(s-m)=\sum_{r=0}^{s+1}(-1)^{s+1-r} U_{p-r}(0)(p-r) !(m-r) ! /\{(m-s-1) !(p-s-1) !(s+1-r) !\} .
$$


If the largest $m$ is small compared with $q$, these methods are not very efficient. In this case, let

$$
T(t)=\sum_{r=0}^{q+1} A_{r}{ }^{q+1-r}, \quad U(t)=\sum_{r=0}^{p} B_{r} t^{p-r},
$$

so that $A_{0}=B_{0}=1$ and

$$
A_{1}=(\vartheta-\kappa)(q+1)+\kappa \sum_{r=0}^{q} \gamma_{r}, \quad B_{1}=p \vartheta+\kappa \sum_{r=1}^{p} \beta_{r}
$$

We have

$$
T(t-m)=\sum_{r=0}^{q+1} A_{r}^{q+1-r} \sum_{l=0}^{r}(-m)^{l}\left(\begin{array}{c}
q+l-r \\
l
\end{array}\right) t^{q+1-r-l} .
$$

With the notation of Jordan [3], let us write $S_{n}^{\delta}$ for the Stirling number of the second kind, so that

$$
S_{n}^{8}=\left[\Delta^{8} t^{n} / s !\right]_{t=0}, \quad S_{n}^{n}=1, S_{n}^{n-1}=\frac{1}{2} n(n-1)
$$

By (13), we have

$$
T_{a+1-s}(-m)=\sum_{r=0}^{g} A_{r} \sum_{l=0}^{8-r}(-m)^{l}\left(\begin{array}{c}
q+1-r \\
l
\end{array}\right) S_{q+1-r-l}^{q+1-s}
$$

and similarly

$$
U_{p-8}(-m)=\sum_{r=0}^{8} B_{r} \sum_{l=0}^{s-r}(-m)^{l}\left(\begin{array}{c}
p-r \\
l
\end{array}\right){S_{p-r-l}^{p-s}}^{-8}
$$

If $m$ is small, these formulae provide a convenient method of calculating the coefficients in (11) for $s \leqslant m$; no others are required. In particular, we can verify (9) and (10) very easily, using (12) and (14).

\section{REFERENCES}

1. W. B. Ford, The asymptotic developments of functions defined by Maclaurin series (Ann Arbor, 1936).

2. H. K. Hughes, Bull. American Math. Soc., 50 (1944), 425-430.

3. C. Jordan, Calculus of finite differences (2nd edn., New York, 1947), 168-179.

4. T. D. Riney, Proc. Amer. Math. Soc., 7 (1956), 245-9.

5. T. D. Riney, Trans. American Math. Soc., 88 (1958), $214-226$. 256.

6. E. M. Wright, J. London Math. Soc., 10 (1935), 287-293; see also correction, ibid 27 (1952),

7. E. M. Wright, Phil. Trans. Roy. Soc., A238 (1940), 423-451 and $A 239$ (1941), 217-232.

8. E. M. Wright, Trans. American Math. Soc., 64 (1948), 409-438.

The Untversity

ABERDEEN 